\title{
'Nca, Signuri, si riccunta di Agatuzza: Agata Messia y otras narradoras sicilianas en las recopilaciones de Giuseppe Pitrè
}

\author{
Marina Sanfilippo \\ Universidad Nacional de Educación a Distancia \\ msanfilippo@flog.uned.es
}

RESUMEN

En este trabajo, después de presentar brevemente a Giuseppe Pitrè y a las narradoras que le contaron los cuentos populares que él publicó, analizo la figura, el repertorio y el estilo de Agatuzza Messia - la mujer que el estudioso palermitano consideró como su «narradora modelo»-, basándome en las narraciones publicadas por Pitrè en I875, en los cuatro volúmenes de las Fiabe, novelle e racconti popolari siciliani, y en I888, en Fiabe e leggende popolari siciliane. Gracias a la cantidad de cuentos de la narradora publicados por Pitrè, a la estrecha relación personal entre los dos y a la sensibilidad y el respecto del estudioso siciliano por aspectos del hecho de narrar que los folkloristas de esa época solían pasar por alto, es posible tener una idea bastante completa de las competencias narrativas y la poética de Agatuzza.

PALABRAS CLAVE

Agatuzza Messia; Giuseppe Pitrè; cuentos populares; narradoras orales; estilo oral

\section{RESUM}

En aquest treball, després de presentar breument Giuseppe Pitré i les narradores que li van explicar les rondalles que ell va publicar, analitzo la figura, el repertori i l'estil d'Agatuzza Messia, la dona que l'estudiós palermità va considerar com la seva «narradora model», basant-me en les narracions publicades per Pitré el I875, en els quatre volums de les Fiabe, novelle i racconti popolari siciliani, i el I888, a Fiabe i leggende popolari siciliane. Gràcies a la quantitat de rondalles de la narradora publicats per Pitré, a l'estreta relació personal entre els dos i a la sensibilitat i el respecte de l'estudiós sicilià per aspectes del fet de narrar que els folkloristes d'aquesta època acostumaven a obviar, és possible tenir una idea bastant completa de les competències narratives i la poètica d'Agatuzza.

PARAules Clau

Agatuzza Messia; Giuseppe Pitrè; rondalles; narradores orals; estil oral 


\begin{abstract}
After a brief introduction of Giuseppe Pitrè and the female storytellers who told him the folk tales that he published, this article analyses the figure, repertoire and style of Agatuzza Messia - the woman regarded by Pitrè as his "model storyteller". The analysis is based on the narrations published by Pitrè in the four volumes of Fiabe, novelle e racconti popolari siciliani in I875 and in Fiabe e leggende popolari siciliane in I888. Thanks to the large number of Messias's tales published by Pitrè, the close relationship between storyteller and the Sicilian scholar, and the sensitivity and respect that Pitrè shows towards certain aspects of narration that other folklorists of the time would often overlook, we now have a fairly complete picture of Agatuzza's narrative and poetic skills.
\end{abstract}

KEYWORDS

Agatuzza Messia; Giuseppe Pitrè; folktales; female storytellers; oral style

REBUT: OI/O9/2OI7 | ACCEPTAT: IO/IO/2OI7 
'Nca, Signuri, si riccunta di Agatuzza: Agata Messia y otras narradoras sicilianas...

\section{Giuseppe Pitrè y las narradoras sicilianas}

\subsection{Un enamorado de la cultura popular}

Mi primer encuentro con Giuseppe Pitrè (Palermo I84I-I9I6) se debe a las páginas de una novela escrita por una mujer, I Daneu. Una familia di antiquari, en la que Alessandra Lavagnino describe a un médico de mirada penetrante y trato amable, que cuenta con gran maestría historias cautivadoras, mientras que ausculta a niñas acatarradas, volviendo así a dar vida a narraciones recogidas anteriormente de los labios de sus pacientes más humildes (2003: 68-69). Poco más tarde descubrí que ese personaje literario había sido en realidad el gran protagonista de la sistematización del folklore como disciplina académica en la Italia de finales del siglo $\mathrm{XIX}^{\mathrm{I}}$ y el primer catedrático de demopsicologia ${ }^{2}$ en la universidad italiana y que sus recopilaciones de cuentos populares «sono più importanti delle fiabe dei Grimm» (Zipes 2012: I69).

Antes de llegar a leer su obra, volví a encontrar a Pitrè en las páginas de unos relatos literarios, transformado en el terrible mago Tre-Pi por el escritor verista Luigi Capuana, que dedicó algunas de sus obras a la creación literaria de cuentos trufados de elementos populares. Este autor fue un gran admirador de la labor de Pitrè, sin embargo encarnó en el mago Tre-Pi los peligros del tipo de postulados teóricos que en esa época se utilizaban para recopilar narraciones tradicionales, fijando sus variantes y separando los etnotextos de su contexto real. ${ }^{3}$

¿Cuál de las dos descripciones se acerca más a la realidad? ¿Un entomólogo que coge esa maravillosa creación efímera que es una narración oral y la colecciona como una mariposa, numerándola y clasificándola, o un hombre enamorado de los cuentos, que los recopila y a veces los vuelve a contar, poniendo así otra vez en circulación lo que recibe? Creo que no peco de excesivo romanticismo inclinándome hacia la segunda opción, por tres razones. En primer lugar, el hecho de que Pitrè aparezca como personaje en narraciones de otros (y a Lavagnino y Capuana hay que sumar Emma Perodi) ${ }^{4}$ coincide con que alrededor de los buenos narradores parecen generarse espontáneamente cierta actividad narrativa; en segundo lugar, existen otras muestras de la capacidad de Pitrè para revitalizar elementos de la cultura tradicional siciliana. ${ }^{5}$ En último lugar, el mismo Pitrè le

I Cf. Cocchiara (1952: 399). Para información sobre Pitrè y su extensa obra, véase también Cocchiara (I98I) y, por lo menos, Fabio Dei (20I5); Benedetti (20I2); Manzo (I999); Gentile (I994) y D'Anna (I993). En el número anterior de esta misma revista publiqué una breve nota informativa sobre el estudioso en ocasión del centenario de su muerte (Sanfilippo 20I6).

2 Para Pitrè, esta palabra corresponde a folklore «per chi in Italia rifugge dai termini stranieri» (Pitrè 200I: 34).

3 El mago conserva los cuentos embalsamados en unos almacenes donde «c'erano tutte le fiabe del mondo, situate nei cassetti fatti a posta, classate e numerate» (Capuana 2006: 197).

4 Gran amiga de Pitrè, que, como cuenta él mismo en una postal a Gennaro Finamore (Toschi 1958: 82), se salvó de un fuerte terremoto en Messina gracias al hecho de haber tenido que ir a Palermo para verla. Ella lo retrató en sus Fiabe fantastiche. Novelle della nonna (1974) como el profesor Luigi, atento recopilador de cuentos tradicionales, capaz de implicarse emocionalmente en la escucha y consciente del valor autorial de una narradora tradicional.

5 Como el hecho de que, gracias a que en i88I el palermitano presentó un carro tradicional siciliano con la «Esposizione industriale de Milano», este objeto destinado a caer en desuso se miniaturizó y dio lugar a una auténtica industria del carrito-souvenir (cf. Manzo I999: 84), todavía viva hoy en día; siempre en este sentido hay también que recordar que, en I895, 
confesó en I9I2 al etnólogo calabrés Raffaele Lombardi Satriani que no estaba seguro de que lo que importaba en el estudio de la narrativa tradicional fueran los aspectos comparativos y de catalogación (Lombardi Satriani I963: IX), mientras que siempre se mostró muy interesado por la narración en sí misma; de hecho, las introducciones y las notas de Pitrè en sus recopilaciones muestran participación, entusiasmo y extraordinaria sensibilidad hacia los narradores y narradoras y hacia el estilo oral de las narraciones. Nunca encontraremos en Pitrè las consabidas críticas hacia las repeticiones, las digresiones y otros supuestos defectos que los recopiladores del siglo xIx y de principios del siglo xx (y en algunos casos hasta mucho más tarde...) achacaban a sus informantes; como escribe Giovanni Gentile, «Pitrè non ebbe mai la pretesa assurda di staccarsi dalla materia dei suoi studi», como entonces predicaban positivistas y veristas (I994: 2I). En lugar de distancia, lo que encontramos en Pitrè es observación participante y emotiva, tanto que la implicación emocional en las narraciones que escuchaba lo lleva a escribir nada menos que a Ernesto Monaci que delante de la narradora Agatuzza «io mi sento annichilito» (Cocchiara I952: 392).

\section{2 «Digo narrador y tendría que decir narradora»}

Agatuzza, la que deja anonadado a Pitrè, era Agata Messia, una narradora que Pitrè define su novellatrice-modello y que es bastante conocida, incluso fuera del ámbito de los y las especialistas, gracias al hecho de que Italo Calvino reprodujo casi integralmente en su antología de cuentos populares italianos (2005: 25-27) la larga descripción que Pitrè le dedicó en el prólogo a Fiabe, novelle e racconti popolari siciliani. ${ }^{6}$ Pero antes de abordar el estudio de Messia, es importante tener claro que, no solo la siciliana pudo contar con un recopilador excepcional sino también que como narradora, se inserta en un contexto de contadoras de historias que comparten cuentos, técnicas narrativas, deseos de independencia femenina y denuncias de abusos e injusticias. ${ }^{7}$

Para empezar, conviene detenerse en el hecho de que Pitrè tuvo muchos informantes, hombres y mujeres; los 458 cuentos de las dos recopilaciones analizadas ${ }^{8}$ en este artículo se deben a I 28 personas adscritas claramente, de una forma u otra,

después de rechazar la posibilidad de ser alcalde de Palermo, Pitrè aprovechó el hecho de ser concejal del ayuntamiento para rescatar y potenciar las antiguas fiestas en honor de Santa Rosalia (Dei 20I5).

6 De aquí en adelante simplemente FNRPS. De sus cuatro volúmenes existe una edición facsímil publicada en 1985 por la editorial Forni y, gracias a la biblioteca digital Liberliber, es posible consultar a una versión de libre acceso en Internet: <https://www.liberliber.it/ mediateca/libri/p/pitre/fiabe_novelle_e_racconti_I/pdf/pitre_fiabe_novelle_e_racconti_I. pdf $>$ [fecha de consulta: septiembre de 20I7]. Cito por la edición bilingüe siciliano-italiano publicada por la editorial Donzelli (Pitrè 20I3).

7 En este sentido, a las narradoras de Pitrè habría que sumar las que aparecen en otra gran recopilación siciliana, la de Laura Gonzembach (I999), con cuentos en los que se habla de incesto y de abusos sexuales.

8 No cuento las variantes que enumera Pitrè al final de cada cuento y respeto la decisión del estudioso de contar como cuentos únicos los ciclos de cuentecillos con un protagonista común (como los de Giufà o Ferrazzano). Si se añadieran las dos cosas se trataría de más de 600 cuentos. 
al género femenino o masculino: 969 hombres y 59 mujeres. A primera vista el número de narradores es algo superior al de narradoras, por lo que sorprende que, en la introducción a FNRPS, Pitrè escriba: «Dico narratore e dovrei dire narratrice, perché le persone da cui ho cercate ed avute tante tradizioni sono state quasi tutte donne» (20I3: I, 9). Sin embargo esta afirmación encuentra su explicación si notamos que, a pesar de ser menos numerosas, fueron mujeres las que contaron más del 60\% de las narraciones recogidas por Pitrè y sus colaboradores ${ }^{\mathrm{Io}}$, y este porcentaje es todavía mayor si consideramos solo los cuentos recopilados directamente por el propio estudioso. A este dato hay que sumar el hecho de que casi todas las narraciones largas y complejas se deben a narradoras y no a narradores, y que, si nos detenemos en las personas que demuestran tener un repertorio de por lo menos seis cuentos, las mujeres son el doble que los hombres (diez frente a cinco). ${ }^{\mathrm{II}}$

Aparte de Agatuzza, Pitrè nos dejó interesantes observaciones sobre otras dos narradoras, Rosa Brusca y Elisabetta Sanfratello. La primera era del Borgo ${ }^{\mathrm{I2}}$ como Messia y le contó muchos cuentos y anécdotas a Pitrè, que la recuerda de la forma siguiente:

Donna sui 45 anni, si rassegna alla sventura della cecità che la colpì, essendo ancora giovane; «tanto, ella dice, che ci guadagnerei a disperarmi?». Prima che perdesse il lume degli occhi era tessitrice (carèra), e le tessitrici contano tra le donne che più sanno canzoni ed anche fatterelli e novelline. La mattina a buona ora presa la sua calza, siede davanti l'uscio di casa, e mentre le mani con moto alterno,continuo, assommano il lavoro, ella tutto brio piacevoleggia e ciarla col vicinato, o motteggia chi passa de' conoscenti, o garrisce il marito, che di quanto guadagna al forno beve altrettanto vino, che gli guasta la testa. Il raccoglimento che gli viene dalla cecità è ragione per cui il suo racconto esce filato, come dice il popolo; onde in lei è talora più minutezzza di circostanze che nella Messia (20I3: I, II-I2).

Elisabetta Sanfratello, la Gnura Sabedda, en cambio, era de Vallelunga ${ }^{\mathrm{I} 3}$ y era una criada de unos amigos de Pitrè, según quien:

La sancta simplicitas de' poveri di spirito è una dote sua particolare, per cui la sua narrazione si fa ingenua. La Sanfratello s'avvicina a' 55 anni e dice di aver appresi i racconti da una sua nonna, che morì a cento. Riferisco

9 En mi recuento dejo fuera los poquísimos casos en los que Pitrè pone solo la inicial del nombre de pila y el apellido, sin ninguna otra indicación que aclare si se trata de una mujer o de un hombre.

Io Todos hombres, entre los que hay que destacar a Salomone Marino, que aportó más de 40 cuentos, y al señor Vincenzo Gialongo, que recogió 24 cuentos de Polizzi generosa, sin señalar por desgracia el nombre de las personas que se los narraron.

II Las narradoras son Francesca Amato, Rosa Brusca, Mara Curatolo, Nina Fedele, Francesca Leto, Ninfa Lobaido, Agatuzza Messia, Angela Puleo, Elisabetta Sanfratello y Angela Smiraglia. Los narradores, Domenico Ingrassia (que con trece cuentos es el hombre que exhibe el repertorio más amplio), Giuseppe La Duca, Antonio Loria, llamado Bonchiàro, Giovanni Patuano y Vincenzo Rappa. Sobre la amplitud de los repertorios narrativos de las mujeres en comparación con los de los hombres, $c f$. Lavinio (20I7: 75).

I2 Barrio popular de Palermo, cercano al puerto.

I3 Pueblecito en la provincia de Caltanissetta. 
qui in nota un tratto caratteristico su questo fatto, e son dolente di non aver avuto tempo di raccogliere colle sue stesse parole il racconto che ella mi fece della sua vita. [Nota I4]: «Sti cunti, signuri, mi li cuntà' mà nanna, e io li cuntu comu mi li cuntà' idda. A Vaddilonga cci ni ajicanu e cci nni prijùncinu; e pricciò nun su' critti... Mà nanna nni sapía assà' di sti cunti... Mà nanna era viacchia, e a fari li cincu vintini (Ioo anni) cci vulianu du' misuzzi. Quandu idda mi li cuntà', io era carusiedda: era tanta..., e idda, la bon'armuzza, mi dicía: “Arrigordatinni la nanna, ca poi quannu si' bedda granni, sti cunti li cunti tu" $»(2013:$ I,I2 $) .{ }^{I 4}$

El repertorio de Brusca publicado por Pitrè se compone de 29 cuentos que pertenecen a géneros distintos (cuentos maravillosos, anécdotas realistas y varios cuentecillos cómicos, algunos protagonizados por el personaje panmediterráneo de Giufà), ${ }^{15}$ mientras que de Sanfratello solo conocemos siete relatos, de los que seis son cuentos maravillosos. Otra narradora cuyo nombre aparece en los prólogos de Fiabe, novelle e racconti popolari siciliani y de Fiabe e leggende popolari siciliane ${ }^{16}$ y de la que Pitrè incluye muchos cuentos en las dos publicaciones es Francesca Amato, una lavandera palermitana de 65 años de edad, que tenía en repertorio por lo menos I9 cuentos, por lo general breves, de corte realista y/o cómico, que en muchos casos vierten sobre la inteligencia o estupidez del/de la protagonista. En cambio, Pitrè no da informaciones de Ninfa Lobaida ni de Francesca Leto, ${ }^{17}$ merecedoras de

I4 Traducción: [Nota I4]: «Estos cuentos, señor, me los contó mi abuela, y yo los cuentos como me los contó ella. En Vallelunga se les añaden cosas sobre la marcha y nunca se acaba de hacerlo y por lo tanto no están escritos...Mi abuela sabía muchos de estos cuentos... Mi abuela era vieja y para cumplir las cinco veintenas le faltaban dos meses. Cuando ella me los contó, yo era pequeña: era así..., y ella, la finadita, me decía: "Acuérdate de la abuela, que después cuando seas bien mayor, estos cuentos los cuentas tú"». Le agradezco a Toti Mercadante la revisión de las traducciones del siciliano.

I5 Sobre el personaje de Giufà en los cuentos sicilianos, $c f$. el estudio de José Manuel Pedrosa (20I0).

I6 De aquí en adelante, FLPS. La edición original está digitalizada: <https://archive.org/ stream/fiabeeleggendepoopitrgoog\#page/nIo/mode/2up > [fecha de consulta: septiembre de 2017]. En I969 la editorial Forni también publicó la edición facsímil de esta recopilación. Cito por la edición bilingüe siciliano-italiano publicada por la editorial Donzelli (Pitrè, 20I6). Es verdad que el estudioso palermitano publicó algunos cuentos de Agatuzza y otros narradores en ediciones anteriores - como por ejemplo las siete Novelline popolari siciliane (1978), publicadas en I874 por el editor Luigi Pedone Lauriel de Palermo en una tirada de tan solo cien ejemplares numerados-, pero volvió a incluirlos casi todos en los tomos de 1875 y I888 sin aportar ningún tipo de modificación en los textos, por lo que las dos recopilaciones de mayor extensión representan el corpus casi completo.

I7 Tampoco comenta nada de María Curatolo, la narradora de tan solo ocho años que le contó un cuento que él juzgó digno de abrir el primer volumen de la recopilación, porque «comincia con mostrare che nelle novelle niente è arbitrario; ma vi sono certe formole [sic] consacrate dall'uso e perpetuate dalla tradizione orale» (2013: I, I54). Sin embargo el interés de Pitrè por este cuento («Lu cuntu de "si raccunta"», es decir «El cuento de "se cuenta"») no se limitaba probablemente a su faceta de reflexión sobre las fórmulas introductorias en la narración oral, sino que su pasado de joven huérfano y de clase humilde, que vio cómo su primer trabajo se esfumaba por culpa de la arrogancia y las mentiras de un superior, debió entrar en sintonía con una narración en la que se celebra la victoria de la inteligencia de una persona pobre e indefensa (doblemente indefensa por su joven edad y su pertenencia al género femenino) frente a la arrogancia de una persona rica, poderosa $y$, al ser hombre y adulto, dotada de mayor autoridad. 
ser recordadas por ser las narradoras de algunos de los cuentos más bonitos de las FNRPS, ${ }^{18}$ pero es probable que esto se deba a que ellas no le contaron sus cuentos directamente a Pitrè, sino a otro médico-folklorista, Salomone Marino, gran amigo y colaborador del primero desde I865 hasta finales del siglo XIX, cuando los dos estudiosos discutieron y dejaron de hablarse (Zipes 20I2: I48).

Gracias al detallismo de las indicaciones de Pitrè, vemos que muchas narradoras ejercían un oficio o una profesión, sobre todo relacionados con tejer y coser —dos actividades que ocupan las manos y sueltan las lenguas...-, por lo que encontramos a modistas, tejedoras, cosedoras, hacedoras de flecos, etc., pero también hay criadas, lavanderas e incluso una mamma del palermitano Conservatorio di S. Agata della Guilla, es decir una mujer que se ocupaba de hacer recados y otras gestiones para mujeres recluidas en monasterios, prisiones $u$ otras instituciones. Quizá sea esta la razón por la que las protagonistas de los cuentos de estas narradoras tienen un oficio o una profesión, algo que suele ser privilegio solo de los protagonistas hombres, mientras que, como subraya Noia (2007: 255), en los cuentos tradicionales las mujeres cuidan de la casa y de la familia, se las describe en relación a los hombres (soltera, casada, viuda...), y la bruja es la única mujer que tiene una profesión, aparte de las tareas domésticas. En las narraciones de las mujeres que contaron para Pitrè y otros colaboradores suyos, encontramos en cambio a comadronas, lavanderas, posaderas, cocineras, dueñas de almacenes de carbón, tenderas, vendedoras, criadas, hilanderas, tejedoras, encajeras, maestras de talleres de coser y, en los cuentos de Agatuzza Messia, vemos a maestras e, incluso, a una directora de una escuela en la que chicos y chicas de distintas clases sociales estudian codo con codo.

\section{Y por fin Agatuzza Messia...}

\subsection{La descripción de Pitrè}

Las observaciones de Pitrè sobre Rosa Brusca y Elisabetta Sanfratello muestran que el folklorista entendió la importancia de temas como el vínculo entre narración y ciertas profesiones, las características de la forma de narrar de las personas ciegas o la importancia de la transmisión intergeneracional del patrimonio narrativo dentro de una misma familia, pero es en la descripción de Agatuzza donde podemos apreciar la modernidad del enfoque del estudioso. Pitrè empieza con un retrato sintético: «Tutt'altro che bella, essa ha parola facile, frase efficace, maniera attraente di raccontare, che ti fa indovinare della sua straordinaria memoria e dello ingegno che sortì da natura» (2OI3: I, XVII), para después ahondar en varios aspectos de la personalidad, la biografía, el repertorio y la forma de narrar de la narradora palermitana. En I874 Agatuzza tenía unos setenta años y sabemos que, cuando Pitrè escribió el prólogo de FLPS en marzo de I888, ella había muerto hacía poco; de ella existe una foto que puede verse en la web del instituto Euroarabo. ${ }^{\text {I9 }}$

I8 Véase, por ejemplo, la narración de Ninfa Lobaido Lu Magu Virgillu (FNRPS LIII), que Pitrè define como «una delle novelle più importanti della mia raccolta» (2OI3: II, 22, nota I) o el relato La soru di lu Conti que contó Francesca Leto y que despertó la admiración de Italo Calvino («La più bella fiaba d'amore italiana, nella più bella versione popolare», 2005: II58).

I9 Cf. Sorgi (20I6): <http://www.istitutoeuroarabo.it/DM/giuseppe-pitre-cento-anni-dopoalcune-considerazioni-sulla-narrativa-di-tradizione-orale/> [fecha de consulta: septiembre 
Su repertorio procedía de su abuela materna, que a su vez lo había recibido como patrimonio narrativo familiar que se remontaba por lo menos a tres generaciones anteriores. Pitrè comenta que Agatuzza lo aprendió de niña y, como tenía buena memoria, ya no lo olvidó. Probablemente, aparte de un repertorio más o menos extenso, de pequeña la palermitana adquirió sobre todo las competencias necesarias para ser una buena narradora, para retener cuentos con facilidad o para saber reconstruirlos, quizá trenzando entre sí distintos relatos o insertando motivos ajenos a la narración original, como se aprecia en varios de sus cuentos recogidos en FNRPS. No sabemos cuántas narraciones conocía, Pitrè publica casi cincuenta cuentos de ella, pero estos podrían corresponder solo a una parte del repertorio de la palermitana, así como los setenta y tres cuentos de Marguerite Philippe que Luzel transcribió representan menos de la mitad de los que la narradora bretona conocía en I870 (Belmont 2007: 13).

Pitrè relata que Agatuzza era una narradora de reconocido prestigio en el barrio y que nadie se cansaba de escucharla. Vivió unos años en Messina y volviendo a Palermo convirtió su estancia en la ciudad del estrecho, tan lejana para el imaginario de sus vecinos, en materia narrativa incluso cómica gracias a su capacidad para imitar otros dialectos. ${ }^{2 \circ}$ Para Pitrè el talento narrativo de Agatuzza se basa en su dominio de la lengua, a pesar de su analfabetismo ( «La Messia non sa leggere, ma la Messia sa tante cose che non le sa nessuno, e le ripete con una proprietà di lingua che è piacere a sentirla», 20I3: I, IO); según él la narradora se valía siempre de las palabras adecuadas para cualquier contexto narrativo. Pitrè señala la importancia de la mímica, los gestos, la postura y la vocalidad de Agatuzza y afirma que sin estos elementos «la narrazione perde metà della sua forza ed efficacia» (20I3: I, II), aunque — nos dice-, por suerte, el lenguaje de Messia está tan inspirado y tiene tal capacidad para dar cuerpo y vida a los detalles de la narración que vale la pena disfrutar de lo que queda. Y también es una suerte para nosotros que el estudioso tuviera una sensibilidad sorprendente para la época para los elementos no verbales y parateatrales de las narraciones, ${ }^{21}$ por lo que a menudo utilizó auténticas acotaciones que, en notas a pie de página, indicaban gestos, mímica y tonos de voz utilizados por el narrador o la narradora. ${ }^{22}$

de 20I7].

20 Para la capacidad de Agatuzza de imitar acentos y dialectos, véase por ejemplo el cuento CCLXV de FNRPS (Pi 'na cipudda di Calàvria si persiru quattru Calavrisi). A veces Messia se lanza incluso a imitar otras lenguas como cuando hace que una princesa-hada diga pugnè y pizzichè (2OI3: II, 40), palabras que según la narradora serían francesas (y significarían puñetazos y pellizcos), o utiliza la expresión nec tibbi nec tabbi, pretendidamente latina (con el significado de no rechistar).

2I Sobre la modernidad de la sensibilidad y los estudios de Pitrè sobre formas espectaculares y parateatrales sicilianas, véase Isgrò (20I7).

22 Valgan de muestra los ejemplos siguientes: «La novellatrice accompagnava questa parola con un gesto ironico, per indicare che il demonio era degno parente del notaio e non già di lei che narrava» (2013: I I64, nota 22, se trata de un cuento de Messia); «Notisi che la narratrice nel nominare Suli, Perna ed Anna facealo con grida lamentose e strazianti» (2OI3: II, 78, nota 23); después de tantu (tanto) «Segna tutto il braccio sinistro, con un gesto bruscamente fatto tra il derisorio e lo scherzevole. Si sa poi che questo allungare il braccio sinistro e segnarne colla mano destra l'estremità più alta, spesso suol farsi in un senso poco delicato ed anche poco onesto» (2OI3: III, I88, nota 5). Por otra parte, si solo los aspectos suprasegmentales podían explicar el sentido de un diálogo o a quién atribuir una frase de la narración, 
Aparte de haber viajado, Messia no era una simple ama de casa, sino que de joven había sido modista y más tarde, cuando ya su vista no era tan buena, se dedicó a coser edredones, es decir, que desempeñó dos oficios que, como ya hemos visto, solían estar vinculados al mundo de la narración. Otro elemento biográfico interesante que Pitrè recuerda es el hecho de que Messia no dejaba pasar un día sin ir a una u otra iglesia a causa de su gran devoción: aparte de la religiosidad de nuestra narradora (que no se refleja especialmente en sus narraciones, ya que los únicos dos miembros del clero que aparecen son un abad corrupto que se apropia de la herencia de un príncipe huérfano y un cura despistado que se olvida de ir a liberar a una chica encerrada, a pesar de que la madre de ella se lo hubiera pedido en su lecho de muerte), ${ }^{23}$ el acudir a iglesias, santuarios y fiestas religiosas era también una forma de relacionarse con una amplia gama de personas, de otros barrios y variadas condiciones sociales, en un lugar y una época poco propicios a la movilidad femenina.

Escribe Michel Valière que «le conteur prend rang de figure emblématique de l'oralité dans le rapport très particulier qui se noue entre lui et son "collecteur" » (20I3), y Pitrè no esconde la familiaridad que existía entre él y su narradora: «La Messia mi vide nascere e mi ebbe tra le braccia» (2OI3: I, II). Es curioso ver que, a pesar de que ella le contó cuentos cuando él era un niño, en las publicaciones de Pitrè casi ninguna de las narraciones de Agatuzza presenta posibles connotaciones infantiles. Pero como también afirma Michel Valière, a veces los «"répertoires" naissent d'une interaction entre les protagonistes de l' "enquête de terrain"» (20I3) y ni a Agatuzza ni a Giuseppe adulto interesarían mucho los escasos cuentos tradicionales dirigidos solo a la infancia.

\subsection{El repertorio de Agatuzza: cuentos, personajes y motivos}

Como sugerí antes, el repertorio de la narradora palermitana no consistió necesariamente solo en los cuentos que publicó Pitrè, ${ }^{24}$ sin embargo, incluso basándonos solo en FNRPS y FLPS, sabemos que Agatuzza no fue una narradora especializada en uno o dos tipos de narraciones, como Amato y Sanfratello, sino que, como Brusca, tuvo un repertorio muy amplio y diversificado; en el que abundan sobre todo los cuentos maravillosos y los cuentos-novela (o cuentos de ingenio, definición que respeta más el valor que Agatuzza atribuía a la inteligencia, humana en general y femenina en particular), pero aparecen también cuentecillos y anécdotas cómicas, algunas leyendas históricas (como la que vierte sobre las

en lugar de modificar el texto, Pitrè, en lugar de añadir algo en el cuerpo de la transcripción, utilizaba igualmente notas a pie de página indicando cómo había que interpretar la frase: «(dimanda il padre alla bambina)» (2OI3: vol. II, IIO, nota 6); «Con queste forme ellittiche la novellatrice riassume tutto il dialogo tra il serpente e Rosina» (2OI3: II, 52, nota I6, se trata de un cuento de Messia); etc. La modernidad de Pitrè se trasparenta también en que en sus notas el estudioso siente la necesidad de recordar en qué situación y para qué público se narró un cuento determinado.

23 Respectivamente en el cuento XI y en el CCXVII de FNRPS. Para la frecuencia con la que la tradición popular siciliana del siglo xIX pone en escena a miembros del clero caracterizados de forma negativa, $c f$. Sorgi (20I6).

24 Aunque no los cite directamente, reconozco que en este trabajo me han acompañado y animado estudios precedentes sobre narradoras como los de De Prada Samper (2004); Milillo (I983) y Tenèze (2000). 
vísperas sicilianas: $c f$. una variante de CCX en FNRPS); varias historias del ciclo leggendario evangelico, como la de Poncio Pilato ni salvo ni condenado (cuento CXIX de FNRPS), la de la madre de San Pedro, condenada al infierno por su maldad irreprimible (cuento CXXVI de FNRPS) ${ }^{25}$ o la de la virgen y los altramuces (cuento XXVIII de FLPS), o narraciones que explican el origen de ciertas costumbres o de algunos proverbios, como la que explica por qué algunas mujeres no se peinan los viernes (XXXVII de FLPS) o las de la prisa de la cucaracha o del asno y el cerdo (CXXI ${ }^{26}$ y $\mathrm{CXXX}^{27}$ de FLPS).

\subsubsection{Personajas de armas tomar}

Hace ya muchos años, Sebastiano Lo Nigro escribía que «il fenomeno che caratterizza veramente la tradizione orale è costituito [...] dalla innovazione, ora felice ora incoerente che ciascun narratore introduce nella forma e perfino nella materia del suo narrare» (I964: 29); para el estudioso, entre los muchos elementos del cuento popular que sufren modificaciones según la personalidad de cada narrador o narradora, destaca el carácter de los personajes, y creo que este aspecto se manifiesta claramente en las narraciones de Agatuzza.

Para lo que se refiere a las y los protagonistas, ${ }^{28}$ la palermitana no habla casi nunca de la belleza ni de hombres ni de mujeres, a no ser que esta sea absolutamente funcional a la historia, y llama la atención la proporción de mujeres activas, independientes y atrevidas que pueblan sus narraciones, sin que padres o maridos puedan o quieran detenerlas; es más, si en varios cuentos el padre de la protagonista le dice explícitamente que le da permiso para que haga lo que quiera («Fa chiddu chi vòi», 2OI3: I, 326, dice por ejemplo el rey, su padre, a la princesa Mandruna), la larga y extraña narración de La panza chi parra —cuento VIII de FNRPS, que según Zipes (Pitrè 20I3: I, 265) no pertenece a ningún tipo narrativo, pero en el que creo que se aprecia una mezcla de ATU 875 y ATU 875B, aderezada por enigmas que acompañan a una genealogía inicial en la que una madre que ayuda a nacer y una abuela que acompaña a los muertos sirven de presentación para una protagonista cuya «barriga que habla» parece casi una reminiscencia del ónfalos del oráculo de Delfos ${ }^{29}$ — se cierra con un rey y marido enamorado que, reconociendo la superioridad intelectual de su mujer, le cede a ella el poder de rei-

25 ATU 804 + ATU 809. Esta mezcla de los dos tipos estaba bastante difundida en Italia incluso en la segunda mitad del siglo xx, tanto que el equipo coordinado por Cirese y Serafini (I975) recogió I9 versiones entre I968 y 1972.

26 ATU 288 B.

27 Corresponde al tipo 207D propuesto por Camarena y Chevalier (I997).

28 En los 48 cuentos de Agatuzza, tres tienen a animales como protagonistas, en los demás 22 protagonistas son hombres y I9 mujeres, aparte de cuatro en los que el protagonismo está compartido. En los cuentos maravillosos las proporciones cambian: tenemos a ocho protagonistas hombres y catorce mujeres, aparte de tres casos mixtos; además antagonistas malvados y ayudantes mágicos tienen a menudo una identidad femenina (véase por ejemplo el cuento de la 'Nfanti Margarita, que corresponde al cuento-tipo ATU 709, es decir, Blancanieves: la chica perseguida se refugia en un palacio en el que es acogida no por enanos o ladrones sino por el alma de una mujer que está purgando sus pecados; Margarita cuidará de la pobre condenada, que a su vez intentará protegerla incluso desde el más allá).

29 Sobre oráculo y sibilas en la antigua cultura siciliana, $c f$. Cocchiara (I978: 46). Entre los cuentos de Agatuzza, el XX de FLPS habla de Sapienza, hermana del rey Salomón. 
nar con unas palabras («Pigghia lu Regnu tu e regna a tò talento, ca tu hai giudiziu pi tia e pi àutru», $3^{\circ}$ 20I3: I, 260), que, en su significado metafórico, siguen representando un cuento maravilloso para muchas mujeres de los cinco continentes...

Encontramos algo parecido en otro de los relatos más representativo del universo de Agatuzza, el cuento VI de FNRPS, Catarina la Sapienti, una variante muy peculiar de ATU 89I, en la que nada sabemos del aspecto físico de la protagonista pero sí que «comu fu smammata cci vinni 'na sapienza ca ogni cosa chi succidia "nta la casa idda avia a dari lu sò disbòtu»" (20I3: I; 220). El padre le deja estudiar todas las lenguas y todos los libros, y cuando ella con i6 años se sume en la tristeza por la muerte de la madre, él sigue el consejo de un grupo de grandes señores y, para distraerla, le ofrece la posibilidad de fundar y dirigir una escuela donde ella misma da clase a cualquiera que lo pida. El príncipe acude a las clases y, como él no sabe contestar a una pregunta, ella le da una bofetada. Él pide casarse con ella pensando en vengarse $y$, puesto que en la noche de boda Catarina no se arrepiente de haberle dado una bofetada al príncipe, el cuento sigue el curso de ATU 89I para terminar felizmente con el marido que se arrepiente pidiendo él perdón, por lo que la pareja desde aquel día puede quererse para siempre. Pitrè recoge también otra versión siciliana que se cierra con la protagonista que declara arrepentirse para salvar así el matrimonio: por lo tanto el final feliz de Agatuzza no era obligatorio en la tradición de la isla, así como no lo era el hecho de que, puesto que en ATU 89I la protagonista engendra a tres hijos en tres ciudades distintas con el mismo príncipe (que por supuesto ignora la identidad de la madre de estos retoños), Catarina se casa otras tres veces con su marido. ${ }^{32}$

Aunque Italo Calvino le achacó a Messia el hecho de que en sus cuentos faltara «lo struggimento amoroso, la predilezione per i motivi dell'amore - sposo o sposa- perduto» (2005: 28), se trata de un juicio erróneo — que sorprendentemente sigue citándose de forma acrítica en estudios actuales-, la realidad es que la larga y penosa búsqueda de novios o maridos perdidos aparece en varios cuentos de Agatuzza, que se adscriben a distintos tipos de ATU 425 (puros o mezclados) como en FNRPS los relatos LVI (Lu sirpenti); XVII (Marvìzia) o XIV (Mandruni e Mandru$n a)$. Lo que falta en las narraciones de Agatuzza son las figuras femeninas pasivas e indefensas, no los personajes de mujeres enamoradas y dispuestas a invertir años y sufrimientos para encontrar al novio perdido. Estas personajas no pierden tiempo en llorar y desesperarse, sino que se inventan estratagemas útiles para descubrir qué le pasó al amado: por ejemplo, la princesa Mandruna, que ha organizado ella sola una fuga de amor con Mandruni, cuando despierta sola en el medio del campo sin saber por qué él ha desaparecido, le pide a un campesino que intercam-

30 Traducción: «Toma tú el reino y reina a tu manera, que tienes juicio [suficiente] para ti y para los demás».

3I Traducción: «en cuanto la destetaron le vino tal sabiduría que tenía que opinar sobre cualquier cosa que pasaba en la casa».

32 De hecho Luisa Rubini, que ha estudiado en profundidad la recopilación de Laura Gonzembach (I999), en una nota al cuento de Sorfarina (muy parecido al de Catarina la Sapienti) considera que la versión de Agatuzza está más censurada que la de Gonzembach, porque la protagonista se casa en lugar de ser amante del príncipe. Creo en cambio que para Agatuzza no se trataba de censura, sino de mostrar que Catarina (sin el apoyo de ningún ayudante mágico, mientras que unas hadas solucionan los problemas de Sorfarina) era capaz de obligar al príncipe a casarse cuatro veces. 
bie con ella la ropa para poder viajar más segura y llega a la ciudad de su amado, donde abre una posada, en la que pone un cartel en el que anuncia que cualquiera que se aloje allí tendrá durante tres días comida y cama gratis, con la condición de que cuente a la posadera toda su vida pasada. ${ }^{33}$ Después de siete años, cuando ella ya no tiene el aspecto de una joven princesa, sino de una arrugada monaca di casa, ${ }^{34}$ aparece Mandruni (que se había perdido persiguiendo un pájaro que le había robado el anillo); su apariencia es la de un pobre mendigo sudado y maloliente, pero Mandruna lo reconoce y, gracias al hecho de que él tiene que contar su vida pasada, descubre que Mandruni no la abandonó voluntariamente. En este punto la narración podría acabar con una fulminante y dichosa agnición, pero Agatuzza decide que su protagonista tiene derecho no solo al amor sino también a un matrimonio sólido y bien aceptado por las familias. La narración por tanto se prolonga hasta que Mandruna no logra el consenso entusiasta de la suegra (que «si la java vasannu cammari cammari», la iba besando de una habitación a otra) y recupera a su padre.

\subsubsection{Que la escritura y la amistad siempre nos protejan}

Agatuzza no sabía escribir pero evidentemente atribuía un gran valor a la escritura y la cultura: en sus cuentos la escritura juega siempre un papel positivo y aparece en múltiples elementos, libros, carteles, cartas y documentos sin que utilice nunca el motivo muy difundido de la escritura portadora de engaños por culpa de cartas falsas o cambiadas. Por ejemplo, Caterina exige que el príncipe reconozca por escrito su paternidad y Mandruna le hace firmar a Mandruni un compromiso matrimonial, que le permite no perder la tranquilidad cuando su futura suegra todavía no está convencida de la oportunidad de una boda ( «Bedda carta mi canta 'n cannolu», 20I3: I, 332). Análogamente el cuento XCII de FNRPS, Lu Principi di Missina, del tipo ATU I407A, se concluye no solo con la usual llegada de testigos que malinterpretan las palabras del protagonista, sino con la aparición de un notario que pone debidamente por escrito las supuestas últimas voluntades del príncipe avaro. ${ }^{35}$ En el cuento Lu Re di Spagna e lu Milordu 'nglisi descubrimos que para Agatuzza aprender a escribir forma parte de la educación de cualquier chica que tenga que casarse con un príncipe (así como el conocer lenguas extranjeras) y de un cuento a otro la escritura representa siempre una ayuda, una ocasión de promoción social (cf. Lu Vicerrè Tunnina) y una protección para héroes y heroínas, sin que falten por supuesto referencias a libros dotados de poderes mágicos ( $c f . L u$ Piscaturi).

Sin embargo, puesto que, desde Homero en adelante, épica, poesía y narrativa -incluidos cuentos maravillosos y populares que ponen el acento sobre madrastras crueles, hermanas envidiosas y pérfidas cuñadas tejedoras de engaños ${ }^{36}$ -

33 El mismo expediente aparece en el cuento de $\mathrm{Lu}$ sirpenti, en el que los viajeros tienen que contar fábulas y cuentos.

34 En la sociedad siciliana de la época, mujeres que podían ser monjas terciarias o simplemente solteras dedicadas al cuidado de iglesias ( $c f$. Pitrè, 20I3: III, I6I).

$35 \mathrm{El} \mathrm{mismo} \mathrm{motivo} \mathrm{aparece} \mathrm{en} \mathrm{una} \mathrm{variante} \mathrm{valenciana} \mathrm{de} \mathrm{este} \mathrm{mismo} \mathrm{cuento} \mathrm{(} c f$. Oriol y Pujol 2003: 298).

36 Hay que reconocer que escapa a la norma el cuento-tipo 5I6D (La doncella serpiente y su amiga leal), propuesto por Camarena y Chevalier en el segundo volumen del catálogo tipo- 
han hablado de la rivalidad femenina dentro y fuera de la familia y de la incapacidad de las mujeres para mantener una amistad, casi negando la existencia de relaciones positivas entre personas de género femenino, quizá el motivo más original en las narraciones de Agatuzza es el insistir en la posibilidad de amistades y alianzas entre mujeres. Messia se rebela con fuerza a la invisibilización de la amistad y la solidaridad femeninas y, por ejemplo, en La mammana di la Principissa-fata (LV de FNRPS) utiliza el molde del cuento-tipo ATU 476 para presentar a una comadrona que no duda en dar de comer a una mano misteriosa que se lo pide y que, como descubre más adelante, pertenece a una princesa-hada embarazada. Para dar a luz, esta última requiere los servicios de la comadrona, que tampoco duda en dejar solo al marido durante un mes para atender a las necesidades de la otra. La mujer recibe a cambio tanto oro como para convertirse en una gran señora para el resto de su vida ${ }^{37}$ y diez años más tarde encuentra otra vez a la princesa, que ha dejado de ser hada y vive con sus hijos en un palacio en el centro de Palermo. ${ }^{38}$ Las dos entablan conversación y la narración acaba escuetamente con la frase "Accussì addivintaru amici pi simpri».39 Agatuzza nos presenta una amistad tan inesperada como interclasista, que quizá refleje la relación privilegiada que ella y Pitrè mantenían, gracias al hecho de que ella lo había tenido entre los brazos cuando él era un niño y de que, en la infancia de Pitrè, la condición social de ambos no era muy distinta. Otros ejemplos inusitados de amistad femenina aparecen en varias narraciones de la palermitana, empezando por la primera de las tres historias que cuenta el papagayo-notario en el cuento II (vol. I: I68-I73): una princesa, que vaga sola por el mundo en búsqueda de su muñeca desaparecida, encuentra a otra princesa prisionera de un brujo ${ }^{40}$ y organiza su rescate. $\mathrm{Al}$ poco de conocerse y antes de lograr derrotar al brujo, las dos jóvenes se tratan como hermanas y juntas se enfrentan al peligro exclamando «o morti tutti dui, o vivi tutti dui!» (vol. I: I7O). ${ }^{4 \mathrm{I}}$ A este propósito, entre los cuentos populares más bellos sobre el valor de la amistad y la fidelidad se encuentran los cuentos-tipo ATU 5I6 (Faithful John) y ATU 5I6C (Amicus and Amelius), cuyos protagonistas son siempre dos hombres; también lo son en Li dui palummi 'nfatati (Las dos palo-

lógico del cuento folklórico español (I995: 454-59) y recogido por Cardigos y Correia (20I5: I, 290-29I, y II, 268-27I), que indican su presencia también en el catálogo griego de cuentos de magia de Georgius Mégas et alii (20I2). Existen versiones nórdicas de este tipo, como el octavo cuento contenido en la recopilación danesa de Svend Grundtvig (I878), en el que la protagonista se transforma en una cierva en lugar de una serpiente (la he encontrado en la traducción al italiano de Bruno Benzi 20I5).

37 «'Unca sta mammana cu sti gran dinari lassò di fari la mammana; misi carrozza, àbbiti javanu e àbbiti vinianu; era 'na signura di li primi di Palermu» (20I3: II, 40. Traducción: «Desde entonces la comadrona con todo ese dinero dejó de trabajar como comadrona; empezó a tener carroza, trajes para aquí, trajes para allí, era una señora de las primeras de Palermo»).

38 Agatuzza pertenece a la familia de narradores y narradoras que, en lugar de hablar de reinos lejanos, se divierten en introducir detalles que contextualizan los cuentos maravillosos en la realidad geográfica más inmediata. En sus cuentos aparecen distintos lugares de Palermo y alrededores.

39 Traducción: «Así se hicieron amigas para siempre».

40 Que tiene amordazada a su prisionera todo el día con un artilugio metálico que solo abre una vez al día para que ella coma; no puedo evitar pensar que el no poder hablar representara para Agatuzza el peor de los tormentos.

4I Traducción: «jo muertas las dos o vivas las dos!».

Estudis de Literatura Oral Popular, núm. 6, 20I7 
mas mágicas) en el que Agatuzza mezcla los dos cuentos-tipo y presenta a los dos protagonistas como un príncipe (Pippinu) y un noble (Gaitaninu) que no solo son hermanos de leche sino que lo son porque sus madres son amigas affiatate (íntimas), por lo que, cuando nacen los niños, la madre de Gaitaninu «manna nni la Riggina e cci dici: "La Principissa nun voli ca a lu Riuzzu lu dassi a nurrizza; la Principissa lu voli nutricari idda: comu nni nutrica unu, nni nutrica dui". La Riggina, tutta cuntenti di sta prupusizioni, cci l'accittò» (20I6: 60). ${ }^{42}$ Por tanto un cuento de amistad viril absolutamente masculino en la mayoría de las versiones está precedido por la amistad y solidaridad entre dos mujeres; además, en la conclusión vuelve a aparecer el elemento femenino, ya que se dice que una vez aclarado el entuerto la mujer de Pippinu trató siempre a Gaitaninu como a un hermano. Poniendo figuras femeninas al principio y al final de la narración, es decir, en esas zonas liminales en la que es más fácil que asomen las creencias y los valores éticos de quien narra, Agatuzza reivindica también para las mujeres los placeres y los deberes de la amistad. También se puede leer desde una perspectiva de género otra desviación del tipo que consiste en contar que Pippinu, para que Gaitaninu resucite, no tiene que matar a su/s hijo/s pequeño/s, un sacrificio que las mujeres no suelen perpetrar, ${ }^{43}$ sino solo a las palomas mágicas que habían desvelado al noble joven los peligros que acechaban a Pippinu con la consigna de que «cu' lu senti e lu cuntirá / tuttu di marmu addivintirá»44 (2016: 64).45

42 Traducción: «hace que le digan a la Reina: "La Princesa no quiere que el Reyecito se lo
den a una nodriza; la Princesa quiere darle de comer ella: como le da de comer a uno, les da
de comer a dos". La Reina, muy contenta por la propuesta, aceptó» (en Sicilia se indica con
príncipe y princesa a un rango de nobleza que no pertenece a la realeza, por lo que el hijo
de un rey no es un príncipe sino un reuzzo, que traduzco aquí como reyecito; la princesa de la
narración de Agatuzza es una dama de corte). 43 Para este rechazo al sacrificio de los hijos, propio no solo de Agatuzza sino del género femenino, véase un ejemplo real recogido por Alessandro Portelli, que relata de una mujer que después de declararse no creyente se refiere a Cristo y «con la rapidissima visione proletaria di una storia sacra possibile, fatta di madri e non di padri "Io s'ero Dio" dice "s'ero lo padre, impicca' no' lo facevo impicca', su la croce” (Portelli 2000: I34. Traducción: «Con la rápida visión proletaria de una historia sagrada posible, hecha de madres y no de padres, "Yo si era Dios" dice "si era el padre, ahorcar no lo dejaba ahorcar, en la cruz"»).

44 Traducción: «quien lo oye y lo cuente /todo de mármol se volverá».

45 Este cuento representa un ejemplo de observación privilegiada de cómo los autores literarios reescriben el material folklórico: la ya mencionada Emma Perodi lo incluyó en su obra Al tempo dei tempi... Fiabe e leggende delle città di Sicilia (publicada en I909), un volumen en el que según la nota editorial previa «l'autrice toscana, che visse a lungo a Palermo e poté raccogliere dalla viva voce del popolo un ricco patrimonio di leggende, coglie nella sua essenza l'arcano dell'isola» (I988) (traducción: «la autora toscana, que vivió mucho tiempo en Palermo y pudo recoger de la viva voz del pueblo un rico patrimonio de leyendas, retrata en su esencia el misterio de la isla»). Puesto que Perodi se trasladó a Sicilia a principios del siglo $\mathrm{xx}$, es difícil que recogiera el cuento directamente de Agatuzza y mucho más probable que conociera las narraciones de la palermitana a través de su amigo Pitrè. Las intervenciones de Perodi tienden a dar una apariencia literaria a la narración ágil y concisa de Agatuzza, pero respetan los nombres de los personajes (Pippinu y Gaitaninu son simplemente italianizados en Giuseppe y Gaetano), las secuencias narrativas y los intercambios dialógicos. Valga como ejemplo la reelaboración de Perodi del fragmento sobre el ofrecimiento de la dama de corte a la reina: «La Principessa appena seppe che al Re era nato l'erede del trono, mandò il marito a dire alla Regina che le concedesse il favore di permetterle che insieme col proprio figlio allattasse il Principe reale. La Regina fu tutta contenta di non farlo allevare da una contadina 
La envidia entre hermanas y cuñadas y la perfidia de las reinas suegras son motivos que a veces aparecen también en las narraciones de Agatuzza, pero no son dominantes sino que la palermitana tiene varios cuentos en los que vemos relaciones de solidaridad y apoyo entre hermanas o cuñadas y a reinas que se ponen del lado de las nueras ( $c f$. la reina madre del cuento Lu Re di Spagna e lu Milord 'nglisi).

\subsection{El estilo narrativo de Agatuzza}

Según Zipes: «Quando ascoltava una storia in dialetto, Pitrè in genere prendeva appunti e, a partire da un ascolto o ancora meglio da due o tre, ricostruiva la narrazione ricorrendo a un metodo misto, in modo da rendere la storia il più possibile comprensibile ai lettori» (2OI3: XXXIII). La larga frecuentación entre el estudioso y su narradora permite pensar que, cuando Pitrè puso negro sobre blanco los cuentos de Agatuzza, no solo los conocía muy bien sino que la voz de ella todavía resonaba en sus oídos. Además algunas notas de FNRPS recuerdan la presencia de otras personas cuando Agatuzza le contaba a Pitrè sus historias, por lo que las narraciones que el folklorista reproduce no eran simples rememoraciones mecánicas, sino que habían tenido un auténtico carácter performativo y Messia había tenido que adaptar a un público concreto el lenguaje del cuento. ${ }^{46}$ Por lo tanto, es probable que en la escritura pitreiana se conserven rasgos importantes de las performances de la palermitana y sea posible rastrear el estilo oral de la narradora, aunque contamos con poco más que la transcripción de lo verbal: la descripción de Pitrè nos autoriza a imaginar cambios de tono, gestualidad rica y variada, hábil manejo de la voz y, además, características textuales, como los cambios de ritmo, las rimas, las comparaciones y los diálogos nos pueden guiar en este análisis.

Entre los elementos que saltan a la vista destaca la variedad de recursos narrativos de Messia; de hecho, las narraciones basan su punto de fuerza en distintos elementos clave, que pueden variar según los cuentos desde los insertos rimados o cantados (véase por ejemplo La viddanedda maritata o la original versión de ATU 89IB* puesta en circulación por Agatuzza: Lu Bracceri di manu manca), hasta la puesta en escena (véase Lu tignusu, lu rugnusu e lu murvusu, un texto muy corto tan

e fece ringraziare la dama e le assicurò che se fosse campata cent'anni, non si sarebbe mai dimenticata di quella gran prova di affetto e cortesia» (I988: 5-6. Traducción: «En cuanto la Princesa supo que al Rey le había nacido el heredero al del trono, mandó a su marido a que le dijera a la Reina que le concediera el favor de amamantar al Príncipe real junto con su propio hijo. La Reina se alegró mucho de no tener que dejar que lo criara una campesina y le mandó su agradecimiento a la dama y le aseguró que aunque viviera cien años no se olvidaría nunca de esa gran prueba de cariño y amabilidad»). No hay espacio aquí para detenerme en un análisis exhaustivo de las razones de los insertos de Perodi, pero creo que salta a la vista de qué tipo de reescritura literaria se trata y, sobre todo, que aun cuando la autora tiene las mejores intenciones y comparte los valores de la narradora (aparte de su interés sincero por los cuentos tradicionales, Perodi, como Agatuzza, tenía una postura feminista ante la vida y era cercana al movimiento de emancipación de las mujeres de la época) «l'intervento della scrittura fa della fiaba un altro oggetto, con il quale condivide solo delle somiglianze superficiali» (Belmont 2002: I69).

46 Como cuando, en presencia de chicas jóvenes, tuvo que explicar el problema de que a la protagonista disfrazada de hombre se le propusiera matrimonio con otra chica y recurrió a la frase «Putía essiri mai lignu con lignu?» (cf. Pitrè 20I3: II, 246, nota 2I. Traducción: «¿Cómo podía funcionar madera con madera?»). 
completamente basado en el talento teatral de Agatuzza que, en una nota, Pitrè tiene que explicar: «Le parole dei tre sono accompagnate dagli atti che essi fanno. La narratrice al primo atto si gratta il capo con tutte e due le mani; al secondo, con la mano destra si gratta l'avambraccio sinistro; al terzo, (il moccioso) struscia il dorso dell'indice destro sotto le narici», 20I6: 570), pasando por el enredo sorprendente, resultado de la mezcla de dos o más cuentos-tipo (véanse cuentos como La panza chi parra; Malvizia; L'arginteri; Grattula-Bedattula, etc.), la parodia de otros dialectos o formas de hablar (Lu viddanu di Larcàra), etc.

De todas formas y no podía ser de otro modo, en muchos aspectos Messia no destaca entre las demás narradoras de Pitrè: utiliza las mismas fórmulas de apertura y cierre de las narraciones, evidentemente formas cristalizadas propias de una tradición compartida y no es ni de las que usan una mayor variedad ni de las que emplean fórmulas especialmente elaboradas o personales. ${ }^{47} \mathrm{Su}$ maestría hay que buscarla más en los elementos no verbales y parateatrales, de los que queda rastro en los largos intercambios dialógicos que salpican sus cuentos: en algunos casos Agatuzza llega hasta los diez u once turnos dialógicos sin necesidad de intercalar demasiados verba dicendi que aclaren quién está hablando; una hazaña narrativa de este tipo es posible solo si quien narra es capaz de instalar sólidamente a los personajes en el espacio y de caracterizar claramente su voz y su forma de hablar. Véase como ejemplo un diálogo entre tres personajes, aderezado además por dos comentarios metanarrativos de Agatuzza, al principio del cuento de Mandruni e Mandruna, cuando la princesa Mandruna ve por primera vez a Mandruni, que trabaja como ayudante en los establos del palacio real (aunque en realidad es un príncipe) y se sorprende por su facci fina (rostro delicado):

Dici la Rigginedda a lu cucchieri maggiuri: «Monsù, cu' è chistu?», «CCillennza —ci arrispunni lu cucchieri-, è un poviru viddanu ca mi lu pigghiai pi muzzu». «Mai, ca chistu, pirsuna bona havi a essere» (dici la Rigginedda). «Veni ccà: (ci dici a lu muzzu), tu dunni si’?». «Di sti paisi» (comu dicissimu di Capaci). «Comu ti chiami?». «Franciscu» (ca iddu s'avia canciatu lu nnomu). «E tò patri chi fa?». «Mulinaru». «E comu lu lassasti?». «CCillenza, mii vulía vastuniari, e io mi nni fujvi». A la Rigginedda stu discursu 'un cci parsi tantu sinceru (Pitrè 20I3: I, 324-326). ${ }^{48}$

El comentario sobre el pueblo de procedencia está dicho como muy de pasada, como si Agatuzza eligiera al azar el nombre del pueblo (comu dicissimu, como si dijéramos, una fórmula que Messia utiliza con frecuencia, siempre con el fin de conectar la narración a la realidad siciliana), sin embargo en una nota a pie de página Pitrè informa que los habitantes de Capaci tenían la fama de ser torpes y tontos, por lo que no solo la narradora estaba dando voz a un largo diálogo, sino que era capaz de insertar notas cómicas añadidas. ${ }^{49}$

47 Para informaciones sobre fórmulas sicilianas de apertura y cierre, $c f$. Sanfilippo (2007).

48 Traducción: «Dice la Princesa al cochero principal: «Jefe, ¿quién es este?», «Su Excelencia -le contesta el cochero-, es un pobre campesino que he cogido como mozo de establo». «QQué dices!, que este tiene pinta de pertenecer a la nobleza» (dice la princesa). «Ven aquí: (le dice al mozo), ¿tú de dónde eres?». «Del pueblo de tal sti paisi» (como si dijéramos Capaci). «¿Cómo te llamas?». «Francisco» (que él se había cambiado el nombre). «¿Y tu padre qué hace?». «Molinero». «¿Y cómo es que lo has abandonado?». «Su Excelencia, me quería apalear y yo me he escapado». A la princesa este discurso no le pareció muy sincero».

49 Agatuzza habla de Capaci también en Cci appizzau lu sceccu e li carrubbi (2013: IV, 272). 
Entre las características estilísticas de Agatuzza creo que sobresale la teatralidad con la que describe las microsecuencias de acción: para seguir con el cuento de Mandruni y Mandruna, cuando el primero persigue el pájaro que ha robado el anillo, Agatuzza lo describe de la forma siguiente: «Chi fa? Si susi, appoja la testa di Mandruna supra 'na trùscia, e si metti a 'ssicutari lu corvu. Curri di ccà, sata di ddà, acchiana, scinni: si spersi senza aneddu e senza Mandruna. Jamu a idda...” (20I3: I, 328)..$^{\circ}$ Es fácil imaginar los movimientos de las manos de Agatuzza que acompañan las acciones del príncipe (corre, salta, sube, baja...), para después dejar la escena vacía (sin anillo y sin Mandruna), volver al punto de partida y mostrar a Mandruna, que se despierta sola, sin anillo, y piensa que el príncipe la ha abandonado, como descubrimos inmediatamente por las frases que pronuncia la chica. De aquí en adelante el cuento corre sobre una doble vía porque Agatuzza tiene que contar lo que le pasa a cada uno de los enamorados separados; lo hace con gran maestría subrayando el cambio con comentarios como «Lassamu a idda e pigghiamu a Mandruni», ${ }^{\mathrm{I}}$ «Turnamu a idda» (2OI3: I, 328)..$^{52}$ Como en el caso de las fórmulas de apertura y cierre, Messia comparte con las demás narradoras estas técnicas de montaje que les permiten seguir tramas paralelas con el simple expediente de dejar o volver a un personaje, por lo general indicando muy claramente la situación en la que se queda el personaje que desaparece de la escena y la del personaje que vuelve a centrar la atención: «Lassamu a lu pappagaddu ch'era 'nta la gàggia e pigghiamu a lu Cavaleri ca si sbattisimava pi vìdiri a la signura» (2013: I, I66, ejemplo de Agatuzza); ${ }^{3}$ "Lassamu a la vecchia chi attacca lu cuani pi guardianu e pigliamu a li cummari» (20I3: I, 408, ejemplo de Sanfratello); «Lassamu a iddu chi torna a la casa e pigghiamu a li ziani, ch'eranu cuntenti» (20I3: I, 596, ejemplo de Vàrrica)..$^{54}$

Para concluir este rápido panorama sobre el estilo de Agatuzza, tres veloces pinceladas sobre aspectos que confirman su maestría narrativa:

I) Como subraya Cristina Lavinio (2007: I05), los mejores narradores manejan con frecuencia un rico repertorio de ideófonos y exclamaciones y Agatuzza confirma la regla no solo con expresiones más o menos corrientes como ih!, vih!, pum!, túffiti (ahí va), sino con onomatopeyas menos comunes como, un ejemplo entre varios, «Tiritinghi e tiritanghi! Nirbati ca jiccavanu focu» (20I6: 208), que se podría traducir, despojándola de su sonoridad, con «iZas por un lado y zas por el otro! Azotes que levantaban centellas».

50 Traducción: «¿Qué hace? Se levanta, apoya la cabeza de Mandruna sobre un fardo y se pone a perseguir al cuervo. Corre por aquí, salta por allá, sube, baja: se pierde sin anillo y sin Mandruna. Vamos a ella...».

5I Traducción: «Dejémosla a ella y cojamos a Mandrone».

52 Traducción: «Volvamos a ella».

53 Sobre como Messia utiliza las funciones de fascinación y dilación del cuento maravilloso en este relato, $c f$. Lavinio (1985: 40-4I).

54 Traducción: «Dejemos al papagayo que estaba en la jaula y cojamos al Caballero que estaba frenético por ver a la señora»; «Dejemos a la vieja que pone al perro de guardia y cojamos a las comadres»; «Dejémosle a él que vuelve a casa y cojamos a los tíos que estaban contentos». 
2) Messia sabe instalar las situaciones narrativas con una rapidez y una economía verbal fulminantes. Por ejemplo, en el ya recordado cuento de las dos palomas mágicas, narra que, después de ser criados por la Principessa, Pippinu y Gaitaninu crecieron como hermanos y estudiaron juntos, hasta que «a l'età di sidici anni, scupetta tutti dui: a caccia!» (20I6: 62):55 con tan solo tres breves enunciados Agatuzza da por concluido el crecimiento de ambos, los saca de la seguridad del palacio, los aísla de los demás y los catapulta solos en el bosque en el que las dos palomas darán inicio al sufrimiento de Gaitaninu.

3) Aprovecha la repetición y la variación mínima a distintos niveles (fónico, léxico, morfológico, etc.) para tejer una narración en la que los ecos sonoros se suceden estableciendo paralelismos entre acciones o elementos. Por ejemplo, la huérfana protagonista di Li tri belli curuni mei! después de huir por los campos entra en un palacio oscuro y misterioso: «Trasi e vidi li gran cammari. Trasi 'nta la cucina e vidi lu beni di Diu. Va nni l'àutri cammari e vidi tutti cosi a gamm'all'aria» (20I3: I: 284; la cursiva y el subrayado son míos). ${ }^{6}$ Subrayado por la reiteración verbal, el crescendo de la visión de grandes salas y grandes riquezas se quiebra en el asombro de encontrar todo gamm'all'aria, patas arriba, que en la oralidad se contrapone fonéticamente a las salas, cammari, que despertaban otro tipo de expectativa.

También las notas de Pitrè en cuentos de otras narradoras son un elemento que ayuda a reconstruir el estilo y la poética de Agatuzza: en este sentido, en el cuento CCXV de FNRPS (narrado por Francesca Campo a Salomone Marino), después de la frase «li cosi caminavanu cu lu verso, no comu caminanu ora, ca li Biati Pauli cci vurrèvanu pi daveru» (20I3: IV, 85), ${ }^{77}$ Pitrè comenta: «Questa qui è un'osservazione come quelle che suol fare Agatuzza Messia», poniendo así de manifiesto el vínculo que su narratrice modello establecía entre el mundo de la ficción y el de la realidad.

\section{Conclusiones}

Es curioso notar que, justo en los años en los que Pitrè recogía los cuentos para su primera gran recopilación, en el norte de Italia algunas intelectuales de clase alta empezaban a pensar que las mujeres tenían que disponer de voz y voto en la sociedad. También la analfabeta Agatuzza, en un Palermo muy lejano del clima cultural y político véneto y lombardo, reivindicaba un mundo en el que no se ningunearan la voluntad y los deseos de las mujeres. No es casual que en sus cuentos aparezcan maestras y que las chicas puedan acudir al colegio: Messia introduce en los cuentos heredados de sus antepasadas un elemento que a ella le tenía que parecer muy positivo y que era propio del momento histórico, ya que, en la

55 Traducción: «A la edad de i6 años, escopeta los dos: ja cazar!».

56 Traducción: «Entra y ve una gran sala. Entra en la cocina y ve una gran riqueza. Va a otra sala y ve todo en desorden».

57 Traducción: «Las cosas iban como tenían que ir, no como van ahora que necesitaríamos de verdad a los Beati Paoli» (se trata de una sociedad de artesanos y gente del pueblo que defendían a la gente humilde de la prepotencia de los poderosos). 
Italia unificada de la segunda mitad del siglo xIX, el Gobierno, convencido del papel que la alfabetización podía jugar en la formación de una nueva identidad nacional, apostó por la escolarización obligatoria, pública y gratuita para niños y niñas y desde el principio el cuerpo docente se caracterizó por una importante presencia femenina.

Quizá para Agatuzza el hecho de contar fue una forma de mejorar su propia vida, asegurándole la compañía y el respeto de un público entusiasta, gracias a su voz inconfundible, su mundo interior y su ironía. Todavía hoy el reflejo escrito de las performances de esta mujer analfabeta, que murió hace aproximadamente I30 años, nos sigue fascinando, gracias también a la escucha respetuosa y cómplice de su mejor oyente, Giuseppe Pitrè.

No hay duda de que Messia, como muchos narradores y narradoras, vinculaba la ficción a la realidad para poder opinar sobre la segunda: era su forma de luchar en favor de un mundo en el que corruptos y prepotentes tuvieran su merecido y las mujeres pudieran ser amadas y respetadas por sus maridos y se lanzaran a construir su destino sin depender de nadie, rebelándose contra la suerte adversa. Es lo que se narra en el cuento de Sfurtuna (LXXXVI de FNRPS), que todavía no había nombrado en este trabajo para poder concluir recomendando leerlo y disfrutarlo, ya que está cuajado de espléndidas personajas que viven de su trabajo y saben negociar sabiamente con las fuerzas que gobiernan el destino. 


\section{Referencias bibliográficas}

Belmont, Nicole (2007): «Elle ne peut lire une seule lettre», Cahiers de littérature orale, n. ${ }^{\circ} 62$ : IO3-I25.

- (2002): Poetica della fiaba. Saggio sul racconto di tradizione orale. Palermo: Sellerio (ed. or. Poétique du conte: essai sur le conte de tradition orale. Paris: Gallimard, I999).

BenedetTi, Amedeo (20I2): "Giuseppe Pitrè nelle lettere agli amici letterati». Lares. Rivista quadrimestrale di studi demoetnoantropologici, n. ${ }^{\circ} \mathrm{LXXVIII} / 3$ (septiembre-diciembre 20I2): 48I-499.

BERni, Bruno (ed. y trad.) (2015): Fiabe dansei. Milano: Imperborea.

CALVINO, Italo (ed.) (2005 [1956]): Fiabe italiane raccolte dalla tradizione popolare durante gli ultimi cento anni e trascritte in lingua dai vari dialetti. I Meridiani. Milano: Arnoldo Mondadori.

Camarena laucirica, Julio; Maxime Chevalier (I995): Catálogo tipológico del cuento folklórico español. Cuentos maravillosos. Madrid: Gredos.

- (I997): Catálogo tipológico del cuento folklórico español. Cuentos de animales. Madrid: Gredos.

CAPUANA, Luigi (ed.) (2006 [1882]): C'era una volta....Palermo: Sellerio.

CArdigos, Isabel; Paulo Correia (20I5): Catálogo dos Contos tradicionais portugueses (com as versões análogas dos países lusófonos), 2 vols. Centro de Estudios Ataíde Oliveira/Edições Afrontamento.

CIRESE, Alberto; Liliana SERAFINI (I975): Tradizioni orali non cantate. Primo inventario nazionale per tipi, motivi o argomenti. Roma: Ministero di Beni culturali e ambientali - Discoteca di Stato. Se puede descargar on line: <http://www. icbsa.it/index.php?it/II4/tradizioni-orali-non-cantate> [fecha de consulta: septiembre de 20I7].

CocchiarA, Giuseppe (I98I): Storia del folklore in Italia. Palermo: Sellerio.

- (I978): Preistoria e folklore. Palermo: Sellerio.

- (I952): Storia del folklore in Europa. Torino: Einaudi.

DeI, Fabio (20I5): «Giuseppe Pitrè». En Dizionario biografico degli Italiani, vol. 84, Roma: Treccani <http://www.treccani.it/enciclopedia/giuseppe-pitre_(Dizionario-Biografico) $>$ [fecha de consulta: julio de 20I7].

D’AnNA, Giuseppe (cur.) (I993): Bibliografia degli scritti di Giuseppe Pitrè (con una aggiunta bibliografica di scritti su Pitrè). Roma: Bulzoni.

Gentile, Giovanni (1994): Giuseppe Pitrè. Palermo: Sellerio.

GRUNDTVIG, Svend (I8I8): Danske folkeœventyr, fundne i folkemunde og gjenfortalte. Kjøbenhaun: C.A.Reitzel.

IsGRÒ, Giovanni (20I7): «La visione scenica di Pitrè e Salomone Marino». Dialoghi mediterranei, n. ${ }^{\circ} 23$ (enero 20I7) <http://www.istitutoeuroarabo.it/DM/ la-visione-scenica-di-pitre-e-salomone-marino/> [fecha de consulta: agosto de 20I7].

LAVAgNino, Alessandra (2003): I Daneu. Una famiglia di antiquari. Palermo: Sellerio. 
'Nca, Signuri, si riccunta di Agatuzza: Agata Messia y otras narradoras sicilianas...

LAVINIO, Cristina (20I7): «Narración oral en femenino». En Marina SANFILIPPO; Helena GuZMÁn; Ana Isabel Zamorano (coords.): Mujeres de palabra. Género y narración oral en voz femenina. Mujer y Literatura. Madrid: UNED, p. 68-86.

- (2007): «Las modalidades del cuento oral y los nuevos narradores». Signa. Revista de la Asociación española de Semiótica, n. ${ }^{\circ}$ I6: 97-I24.

- (I985): «Potenza e magia della fiaba. La fiaba tra i generi della prosa narrativa orale tradizionale». La ricerca folklorica, n. ${ }^{\circ}$ I2 (octubre I985): 37-48.

LOMBARDi SATRIANI, Raffaele (I963): Racconti popolari calabresi, vol. IV. Cosenza: Casa del Libro.

Lo Nigro, Sebastiano (I964): Tradizione e invenzione nel racconto popolare. Firenze: Leo S. Olschki.

MANZO, Pasqualina (I999): Storia e folklore nell'opera museografica di Giuseppe Pitrè. Frattamaggiore (Napoli): Edizioni Istituto di Studi Atellani.

Milillo, Aurora (I983): La vita e il suo racconto tra favola e memoria storica. Roma-Reggio Calabria: Casa del Libro.

NoiA CAMPOS, Camiño (2007): «Figuras de muller na narrativa popular». En Helena González y M. Xesús Lama (eds.): Actas do VII Congreso Internacional de Estudos Galegos. Mulleres en Galicia. Galicia e os outros pobos da Península. Sada: Ediciós do Castro/AIEG/Filoloxía Galega (Universitat de Barcelona), p. 253-266.

ORIOL, Carme (2OI7): «Cuentos populares con protagonistas activas. La cara más desconocida de la tradición». En Marina SANFILIPPo; Helena GuZmán; Ana Isabel ZAMORANO (coords): Mujeres de palabra. Género y narración oral en voz femenina. Mujer y Literatura. Madrid: UNED, p. I7-32.

OrIOL, Carme; Josep M. Pujol (2003): Índex tipològic de la rondalla catalana. Barcelona: Generalitat de Catalunya.

Pedrosa, José Manuel (20Io): «Giufà, Yojá, Nasreddin Hoca, el mulá Nasreddin... o las metamorfosis del trickster». En Romina Reitano; José Manuel PEDrosA: Las aventuras de Giufà en Sicilia. Cabanillas del Campo (Guadalajara): Palabras del Candil, pp. I6-40.

Perodi, Emma (I988 [1909]): Al tempo dei tempi... Fiabe e leggende delle Città di Sicilia. Firenze: Salani.

— (I974 [1893]): Fiabe fantastiche. Le novelle della nonna. Torino: Einaudi.

PITRÈ, Giuseppe (20I6 [I888]): Fiabe e leggende popolari siciliane. Edizione integrale in dialetto siciliano con testo italiano a fronte. Traduzione e cura di Bianca LAzZAro. Prefazione di Giovanni Puglisi. Nota critica di Jack Zipes e appendice su La leggenda di Cola Pesce. Roma: Donzelli.

— (20I3 [I875]): Fiabe, novelle e racconti popolari siciliani, 4 vols. Edizione integrale in dialetto siciliano con testo italiano a fronte. Traduzione di Bianca Lazzaro. Introduzione e cura di Jack Zipes. Prefazione di Giovanni Puglisi. Roma: Donzelli.

- (200I): La demopsicologia e la sua storia, a cura di Loredana Bellantonio. Comiso (RG): Ministero per i Beni e le Attività culturali, Centro Internazionale di Etnostoria, Documenta Edizioni. 
PITRÈ, Giuseppe (I978 [I874]): Novelline popolari siciliane. Raccolte e annotate da Giuseppe Pitrè, tradotte da Sebastiano ADDAMo, Giuseppe BonAVIRI, Vincenzo Consolo, Leonardo Sciascia. Illustrate da Bruno Caruso. Con una introduzione di Italo Calvino. Palermo: Sellerio.

PORTElli, Alessandro (200o): «Un lavoro di relazione. Osservazioni sulla storia orale». Ricerche storiche salesiane, n. ${ }^{\circ}$ XIX/I: I25-I34.

PradA SAMPER, José Manuel de (2004): El pájaro que canta el bien y el mal. La viday los cuentos tradicionales de Azcaria Prieto (I883-I970). Madrid: Lengua de Trapo.

SANFILIPPO, Marina (2007): «Si cunta e s'arriccunta: las fórmulas de apertura y cierre en la narración oral». Revista de dialectología y tradiciones populares, n. ${ }^{\circ}$ LXII/2 (julio-diciembre 2007): I35-I63.

— (20I6): «Sobre Giuseppe Pitrè en el centenario de su muerte». Estudis de Literatura Oral Popular/Studies in Oral Folk Literature, n. ${ }^{o}$ 5: I45-I57. DOI: http://dx. doi.org/IO.I7345/elop20i6I45-I57

- (20I7): «Agatuzza e le altre. Donne e racconto orale». En M. S. SAPEGNO; I. DE Bernardis; A. PERrotta (eds.): Critica clandestina? Studi letterari femministi in Italia. Roma: Sapienza Università editrice, p. I75-I87.

SoRGI, Orietta (20I6): «Giuseppe Pitrè, cento anni dopo. Alcune considerazioni sulla narrativa di tradizione orale». Dialoghi mediterranei, n.o 20 (julio 20I6) $<$ http://www.istitutoeuroarabo.it/DM/giuseppe-pitre-cento-anni-dopo-alcune-considerazioni-sulla-narrativa-di-tradizione-orale/> [fecha de consulta: julio de 2OI7].

TÉNÈze, Marie-Louise; Georges Delarue (eds.) (200o): Nannette Lévesque conteuse et chanteuse du pays des sources de la Loire. Paris: Gallimard.

TosCHI, Paolo (I958): «Fabri» del folklore. Ritratti e ricordi. Roma: Angelo Signorelli.

UTHER, Hans-Jörg (2004): The Types of International Folktales. A classification and Bibliography. Based on the System of Antti Aarne and Stith Thompson. 3 vols. Folklore Fellows' Communications 284, 285, 286. Helsinki: Suomalainen Tiedeakatemia.

VALIÈre, Michel (20I3): «L'invention du conteur et la vocation du collecteur». Ethnographiques.org, n. ${ }^{\circ} 26$ (julio 2013) <http://www.ethnographiques. org/20I3/Valiere $>$ [fecha de consulta: agosto de 20I7].

ZIPES, Jack (2OI2): La fiaba irresistibile. Storia culturale e sociale di un genere. Trad. de Marco Giovenale. Roma: Donzelli (ed. or. The Irresistible Fairy Tale. The Cultural and Social History of a Genre. Princeton University Press, 20I2).

— (20I3): «Il mondo raccontato dal popolo: la straordinaria avventura di Giuseppe Pitrè». En FNRPS, p. XVII-XLI. 